Research Article

\title{
The Role of T Helper 17 (Th17) and Regulatory T Cells (Treg) in the Pathogenesis of Vulvovaginal Candidiasis among HIV-Infected Women
}

\author{
Teke Apalata $\mathbb{D}^{1,2}$ and Benjamin Longo-Mbenza $\mathbb{D}^{3,4}$ \\ ${ }^{1}$ Division of Medical Microbiology, Department of Pathology \& Laboratory Medicine, Faculty of Health Sciences, \\ Walter Sisulu University, Private Bag X1, Mthatha 5117, Eastern Cape, South Africa \\ ${ }^{2}$ National Health Laboratory Services (NHLS), Nelson Mandela Academic Hospital, Mthatha, South Africa \\ ${ }^{3}$ Faculty of Health Sciences, Walter Sisulu University, Private Bag X1, Mthatha 5117, Eastern Cape, South Africa \\ ${ }^{4}$ Department of Internal Medicine, Faculty of Medicine, University of Kinshasa, Kinshasa, Democratic Republic of the Congo
}

Correspondence should be addressed to Teke Apalata; ruffinapalata@gmail.com

Received 18 July 2020; Accepted 28 October 2020; Published 12 November 2020

Academic Editor: Sujata Prasad

Copyright (c) 2020 Teke Apalata and Benjamin Longo-Mbenza. This is an open access article distributed under the Creative Commons Attribution License, which permits unrestricted use, distribution, and reproduction in any medium, provided the original work is properly cited.

Background. The study sought to describe relationships between 20 cytokines and chemokines (IL- $1 \beta$, IL-2, IL-4, IL-5, IL-6, IL7, IL-8, IL-10, IL-12, IL-13, IL-17, G-CSF, GM-CSF, IFN- $\gamma$, MCP-1, MIP- $1 \beta$, TNF- $\alpha$, TGF- $\beta 1$, TGF- $\beta 2$, and TGF- $\beta 3$ ) and the presence of vulvovaginal candidiasis (VVC) in women, stratified by HIV status. Methods. Plasma and genital samples were obtained from 51 clinic attendees in KwaZulu-Natal between June 2011 and December 2011. Cytokine and chemokine concentrations were measured by Luminex ${ }^{\circledR}$ multiplex immunoassays. Multiple comparisons of means of cytokine/chemokine levels displaying significant differences in univariate analyses across the study groups were performed using post hoc Bonferroni pairwise tests considering a type I error rate of 0.05. A discriminant analysis (DA) was carried out to identify linear combinations of variates that would maximally discriminate group memberships. Results. Of the 51 participants, $16 / 26 \mathrm{HIV}$ infected and 15/25 HIV-uninfected women were diagnosed with VVC. DA identified 2 variables (MIP-1 $\beta$ and TGF- $\beta 3$ ) in plasma (Box's M (5.49), $p(0.57)>\alpha(0.001)$; Wilks' lambda $=0.116, p<0.0001)$ and 1 variable (IL-13) in vaginal secretions (Box's M (2.063), $p(0.37)>\alpha(0.001)$; Wilks' lambda $=.677, p<0.0001)$ as able to discriminate the HIV + VVC + group, whilst TGF- $\beta 1$ in plasma discriminated the HIV + VVC - group. Mean concentrations of genital IL-6, IL- 8 , IL- 10 , IL- 17 , and TGF- $\beta 3$ were significantly higher in HIV infected women coinfected with VVC. Conclusions. In HIV-infected women, VVC might be explained by a decline of Th17 cells, hence a decrease of Th17/Treg ratio.

\section{Introduction}

In HIV-infected patients, de Repentigny et al. in 2004 and Fidel Jr. in 2006 reported on the role played by mucosal and cell-mediated immunity against both oral and oesophageal Candida infections $[1,2]$. Unfortunately, the systemic cellmediated immune response was found to be of no protective value against vulvovaginal candidiasis (VVC) as demonstrated by in vitro studies and animal models [3]. Studies have shown that TGF- $\beta$, a well-known downregulatory cytokine, is constitutively expressed in the mucosal surfaces of the vagina independently of the presence of Th1 specific anti-Candida response $[3,4]$.

A study by Shacklet and Anton in 2010 established the presence of Th17 as a subset of CD4+ T cells, which are able to secrete IL-17 and IL-22. The authors have underlined that these secreted cytokines induce the occurrence of peptides with anti-Candida activity, hence having the potential to inhibit the development of VVC [5]. It is not well elucidated whether or not the action of Th17 cells is reduced due to CD4+ $\mathrm{T}$ cells depletion observed during advanced HIV infection, leading to recurrent episodes of VVC. What is 
known is that numerous HIV particles are further expressed through toll-like receptors when infected leukocytes (neutrophils and macrophages) are attracted into the vaginal mucosa following the initial HIV infection [6, 7]. It is however not well understood, whether the secreted IL-17 and IL-22 interfere with the activity of the polymorphonuclear (PMN) cells and macrophages and whether this interference facilitates the development of VVC.

The present study sought to quantify plasma and genital cytokines/chemokines associated with increased susceptibility for vulvovaginal candidiasis and to determine which cytokines/chemokines discriminate between HIV-infected and HIV-uninfected women with and without VVC.

\section{Materials and Methods}

2.1. Study Participants (Patients). During a cross-sectional study, which enrolled 200 black African women between June and December 2011, presented each with a presumptive diagnosis of lower genital tract infection (LGTI), only 51 women were finally eligible for participation into this present study after signing a written informed consent $[8,9]$. The study was conducted in Umlazi D Clinic-a primary healthcare center (PHC) in the South African province of KwaZulu-Natal. Inclusion criteria into the study included age of 18 years and above as well as the presence of symptoms such as vaginal discharge, hypogastric pain, vulval itching, and soreness, as well as dyspareunia. Signs included vulval erythema with or without oedema, excoriations, and/or the presence of thick curdy vaginal discharge. Exclusion criteria included patients' age below 18 years and presence of visible blood in genital area including menses [8]. Commonest causes of LGTIs were screened from genital samples using various microbiological and molecular tests as published elsewhere $[8,9]$. Briefly, Chlamydia trachomatis and Neisseria gonorrhoeae were tested from cervical swabs using a strand displacement amplification technology (Becton Dickinson Probetec Assays, Sparks, Maryland, USA) [9]. QIAamp DNA mini kits (Qiagen Ltd., Chatsworth, CA) were used to extract DNA of Mycoplasma genitalium, Trichomonas vaginalis, and herpes simplex virus type 2 followed by amplification techniques using in-house PCR method and analysis of the amplicons by gel electrophoresis as previously described [9]. Nugent's score, following Gram stain performed on smears made from vaginal swabs obtained from women's anterior fornix, was used to exclude the presence of bacterial vaginosis (BV), when the score was from 7 to $10[8,9]$.

The 51 women retained in this present study were eligible because they were free from Chlamydia trachomatis, Neisseria gonorrhoeae, Mycoplasma genitalium, Trichomonas vaginalis, herpes simplex virus type 2 , and bacterial vaginosis, the commonest causes of nonulcerative LGTIs in women. Of the 51 women, 16/26 HIV infected and 15/25 HIV uninfected women were diagnosed with symptomatic VVC as described in the section below, but free of other causes of LGTIs. Additional patients' characteristics were published elsewhere [9]; of the $26 \mathrm{HIV}$ infected women, 11 (42.3\%) had CD4+ T cell count $<200$ cells $/ \mathrm{mm}^{3}$ (of which 8 patients had plasma viral load $\geq 4 \log _{10}$ copies/mL and 3 had plasma viral load between $1.3 \log _{10}$ and $3.99 \log _{10}$ copies/mL) and $15(57.7 \%)$ had CD4+ T cell count between 200 and 349 cells $/ \mathrm{mm}^{3}$ (of which 14 patients had plasma viral load between $1.3 \log _{10}$ and 3.99 $\log _{10}$ copies/mL and 1 patient had plasma viral load $<1.3$ $\log _{10}$ copies/mL). All HIV-infected patients were receiving regimen 1 antiretroviral therapy (ART) made of two nucleoside reverse-transcriptase inhibitors (NRTIs) and one nonnucleoside reverse-transcriptase inhibitor (NNRTI). None of these patients received a protease inhibitor (PI). The mean duration ( \pm standard deviation) on ART for $25 / 26 \mathrm{HIV}$ positive patients was $4.2( \pm 1.6)$ months. The remaining 1 patient with undetectable plasma viral load $\left(\mathrm{VL}<1.3 \log _{10}\right.$ copies/mL) received ART for 10.7 months. Of $26 \mathrm{HIV}$-positive women, all 11 with CD4+ T cells $<200$ cells $/ \mathrm{mm}^{3}$ and 5 with CD4+ T cells between 200 and 349 cells $/ \mathrm{mm}^{3}$ had confirmed symptomatic VVC. T cells for HIV negative patients were not measured.

\subsection{Diagnostic Criteria of Symptomatic Vulvovaginal Can-} didiasis ( VVC). From each of the eligible 51 study participants, vaginal swab (Becton Dickinson) was taken from the anterior fornix and subsequently plated onto Sabouraud Dextrose agar with chloramphenicol $\left(\mathrm{BBL}^{\mathrm{TM}}\right.$ Becton Dickinson) and incubated at $29^{\circ} \mathrm{C}$ for $48 \mathrm{~h}$. The numbers of yeast colonies per plate were determined and the 2011 European guidelines were applied for the final diagnosis of symptomatic VVC $[8,10,11]$. Briefly, the absence of symptomatic VVC was established when women whose genital specimens were collected showed negative microscopy and culture results for Candida or had microscopy negative with less than 10 colonies of Candida per plate [11].

The identification of the Candida isolates at species level as well as the antifungal susceptibility testing of these cultured isolates was not the focus of this study.

\subsection{Specimen Collection and Measurement of Cytokines/ Chemokines. Blood tubes containing ethyl-} enediaminetetraacetic acid ( $\mathrm{K}_{2}$ EDTA, Becton Dickinson) and non-EDTA reagents were used for the collection of blood samples by venipuncture. A vaginal tampon, Tampax Regular $^{\circledR}$ (Compak), was used to collect vaginal fluid and then subsequently placed into a sterile container with $10 \mathrm{~mL}$ of phosphate buffered saline (PBS; Oxoid Limited Basingstoke, $\mathrm{UK})(\mathrm{pH}=6.9)$, stored at $4^{\circ} \mathrm{C}$ for a period of 4 hours prior to transport to the laboratory. Tampon containing fluid was expressed by means of an autoclaved wooden tongue depressor and, thereafter, filtered using a $0.22 \mu \mathrm{m}$ cellulose acetate filter membranes (Sigma) [8].

A Luminex multiplex assay using fluorescent bead-based immunoassay techniques (Bio-Rad Laboratories, Inc., Parkwood) was used to quantify concentrations (in $\mathrm{pg} / \mathrm{ml}$ ) of IL-1 $\beta$, IL-2, IL-4, IL-5, IL-6, IL-7, IL-8, IL-10, IL-12, IL-13, IL-17, G-CSF, GM-CSF, IFN- $\gamma$, MCP-1, MIP-1 $\beta$, TNF- $\alpha$, TGF- $\beta 1$, TGF- $\beta 2$, and TGF- $\beta 3$ as previously reported [8]. The limit of detection $(\mathrm{pg} / \mathrm{mL})$ was as follows: IL- $1 \beta(0.6)$, IL-2 (1.6), IL-4 (0.7), IL-5 (0.6), IL-6 (2.6), IL-7 (1.1), IL-8 (1.0), IL-10 (0.3), IL-12 (3.5), IL-13 (0.7), IL-17 (3.3), G-CSF 
(1.7), GM-CSF (2.2), IFN- $\gamma$ (6.4), MCP-1 (1.1), MIP-1 $\beta$ (2.4), TNF- $\alpha$ [6], TGF- $\beta 1$ (1.7), TGF- $\beta 2$ (14.7), and TGF- $\beta 3$ (2.8); and a 5 PL regression formula was used to calculate cytokine/chemokine concentrations from the standard curves (Bio-Plex Manager software, version 4). Values below the lower limit of detection were reported as the midpoint between the lowest concentrations measured for each cytokine and zero [8].

2.4. Statistical Analysis. Means \pm standard error of the mean (SEM) and proportions (percentages) were used to summarize continuous and categorical variables, respectively. Analysis of the variance (ANOVA) differentiated between groups for normally distributed data whilst Mann-Whitney $U$ or Kruskal-Wallis nonparametric tests were used for asymmetrically distributed data. Multiple comparisons were performed using post hoc Bonferroni pairwise tests with a $p$ value of less than 0.05 being considered significant. Canonical discriminant analysis (DA) was used to discriminate group memberships and Mahalanobis distances were used for supporting the classification of variates into distinct groups, with values of Wilks' lambda closer to zero being the evidence for well separated groups. In DA analysis, each variate that was more than 1.96 Mahalanobis distance units from the centroid group had a less than $5 \%$ chance of belonging to that group. Box's Test of Equality of Covariance Matrices (with $p<0.001$ ) was used to ascertain the assumption of group homogeneity. Data analysis was performed using SPSS ${ }^{\circledast}$ statistical software version 21.0 (SPSS Inc., Chicago, IL).

\section{Results}

3.1. Concentrations of Plasma Cytokines/Chemokines Associated with Increased Odds for Symptomatic Vulvovaginal Candidiasis in HIV-Infected and Uninfected Women. In univariate analysis, Table 1 depicts the mean concentrations of all plasma cytokines/chemokines that displayed significant differences across the 4 groups. Five potential predictor variables (IL- $1 \beta$, MIP- $1 \beta$, TGF- $\beta 1$, TGF- $\beta 2$, and TGF- $\beta 3$ ) were identified by ANOVA as meeting the initial DA assumption of being normally distributed (Table 1). Higher mean levels of plasma IL- $1 \beta$, TGF- $\beta 1$, and TGF- $\beta 2$ were significantly observed in HIV + VVC- group whilst higher mean levels of plasma MIP- $1 \beta$ and TGF- $\beta 3$ were seen in HIV + VVC + group.

After adjusting for multiple comparisons, those 5 potential predictor variables were confirmed to display significant differences across the study groups using post hoc Bonferroni pairwise tests (Figure 1): the HIV + VVC + group had a significantly lower mean level of IL- $1 \beta$ compared to the mean level of the HIV + VVC- group $(p<0.0001)$ and the HIV-VVC- group $(p=0.023)$, suggesting that lower concentrations of IL-1 $\beta$ were strongly associated with symptomatic VVC in HIV-infected women $(p=0.0003)$ (Figure 1(a)).

When comparing the mean levels of MIP- $1 \beta$, the HIV-VVC- group had a significantly lower mean concentration than the mean value of the HIV + VVCgroup $(p=0.012)$ and more importantly of the HIV + VVC + group $(p<0.0001)$, suggesting that lower concentrations of MIP- $1 \beta$ were strongly associated with the absence of symptomatic VVC in HIV-uninfected women $(p=0.0004)$ (Figure 1(b)). The highest mean concentration of TGF- $\beta 1$ was observed in the HIV + VVC- group as compared to the mean level of every other group $(p=0.0001)$ (Figure 1(c)). A significant difference in mean levels of TGF- $\beta 2$ was observed when comparing the HIV-VVC- group with the HIV-VVC + group $(p<0.006)$ and also between the HIVVVC- group and the HIV + VVC- group $(p=0.002)$ (Figure $1(\mathrm{~d}))$. The mean level of TGF- $\beta 3$ was significantly higher $(p=0.013)$ in the $\mathrm{HIV}+\mathrm{VVC}+$ group and also in the HIV-VVC + group as compared to the mean level of every other remaining group $(p=0.005)$, suggesting that higher levels of TGF- $\beta 3$ were strongly associated with symptomatic VVC irrespective of HIV serostatus (Figure 1(e)).

Canonical discriminant analysis (CDA) was carried out with normally distributed variables that showed significant univariate associations. CDA identified $3 \mathrm{ca}-$ nonical functions and 3 significant variates (MIP- $1 \beta$, TGF$\beta 1$, and TGF- $\beta 3$ ) able to discriminate between the 4 study groups.

The obtained 3 discriminant functions had eigenvalues of $3.516,0.864$, and 0.022 with canonical correlations of $0.882,0.681$, and 0.147 , respectively. The larger eigenvalue represents more shared variance in the linear combination of variables. Of the 3 identified canonical functions, the first 2 functions contributed a total of $99.5 \%$ of the total variance, which easily stratifies into the 4 study groups (HIV-VVC-; HIV-VVC+; HIV + VVC; and HIV + VVC+).

The multivariate Box's $\mathrm{M}$ test was used and confirmed that variables followed a multivariate normal distribution, and the variance-covariance matrices were equal (homogeneity of variances/covariances) across the 4 study groups (Box's M (5.49), $p(0.57)>\alpha(0.001)$ ). Wilks' lambda value was significant: $0.116 ; \chi 2=95.78 ; p<0.0001$ (Table 2).

Functions' coefficients were computed and used in deciding which variable predicted group membership. Comparing the values between groups, the higher coefficient means the predictor variable is attributed more to that group (Table 3 ). TGF- $\beta 1$ strongly predicted HIV + VVC- group while TGF- $\beta 3$ and MIP- $1 \beta$ predicted HIV + VVC + group as shown in Table 3.

Standardized canonical discriminant function coefficients were used for predicting functions defining group centroids (Figure 2). The canonical group means (also called group centroids) are the mean for each group's canonical observation scores. The larger the difference between the canonical groups, the better the predictive power of the canonical discriminant function in classifying observations.

Predictor variables maximizing Mahalanobis distances between two closest groups are depicted in Table 4 .

Taking into account that each variate that was more than 1.96 Mahalanobis distance units away from a specific centroid group had a less than $5 \%$ chance of belonging to that group, from Table 4 , it can be concluded that MIP- $1 \beta$ was 
TABLE 1: Comparisons of the plasma mean levels (in $\mathrm{pg} / \mathrm{ml}$ ) of cytokines and chemokines across the study groups by HIV/VVC status.

\begin{tabular}{|c|c|c|c|c|c|c|}
\hline & & \multicolumn{5}{|c|}{ Study groups } \\
\hline & & $\begin{array}{l}\text { HIV- and VVC- } \\
\text { mean } \pm \text { SEM }\end{array}$ & $\begin{array}{l}\mathrm{HIV}-\text { and VVC+ } \\
\text { mean } \pm \text { SEM }\end{array}$ & $\begin{array}{c}\mathrm{HIV}+\text { and } \mathrm{VVC}+ \\
\text { mean } \pm \text { SEM }\end{array}$ & $\begin{array}{c}\mathrm{HIV}+\text { and VVC- } \\
\text { mean } \pm \text { SEM }\end{array}$ & $\begin{array}{l}\text { ANOVA } p \text { value } \\
\text { (unadjusted) }\end{array}$ \\
\hline Cytokine types & \multicolumn{6}{|c|}{ Cytokine limit of detection (pg/mL) } \\
\hline \multicolumn{7}{|c|}{ Proinflammatory } \\
\hline IL- $1 \beta$ & 0.6 & $4.01 \pm 0.70$ & $3.04 \pm 0.60$ & $5.71 \pm 0.01$ & $2.07 \pm 0.21$ & $<0.0001$ \\
\hline MIP-1 $\beta$ & 2.4 & $68.11 \pm 1.17$ & $128.86 \pm 17.07$ & $158.24 \pm 0.01$ & $182.20 \pm 22.85$ & $<0.0001$ \\
\hline G-CSF* & 1.7 & $0.85 \pm 0.00$ & $3.43 \pm 1.81$ & $4.44 \pm 0.002$ & $2.16 \pm 1.35$ & $0.038^{*}$ \\
\hline GM-CSF* & 2.2 & $48.12 \pm 0.76$ & $30.04 \pm 9.27$ & $1.10 \pm 0.00$ & $88.18 \pm 58.75$ & $0.018^{*}$ \\
\hline IFN- $\gamma^{*}$ & 6.4 & $28.02 \pm 9.33$ & $96.66 \pm 73.35$ & $3.20 \pm 0.00$ & $10.60 \pm 4.35$ & $0.032^{*}$ \\
\hline IL-2* & 1.6 & $3.07 \pm 1.02$ & $18.93 \pm 13.06$ & $0.8 \pm 0.00$ & $0.8 \pm 0.00$ & $0.003^{*}$ \\
\hline IL-8** & 1 & $6.05 \pm 1.092$ & $7.16 \pm 0.94$ & $9.79 \pm 0.001$ & $7.07 \pm 0.91$ & $0.015^{* *}$ \\
\hline TNF- $\alpha^{* *}$ & 6 & $27.31 \pm 7.38$ & $24.23 \pm 17.07$ & $6.86 \pm 0.001$ & $3.28 \pm 2.31$ & $<0.0001 * *$ \\
\hline $\mathrm{MCP}-1^{* *}$ & 1.1 & $101.15 \pm 2.05$ & $100.33 \pm 25.11$ & $70.88 \pm 0.001$ & $75.92 \pm 11.11$ & $0.075^{* *}$ \\
\hline IL-6* & 2.6 & $19.24 \pm 4.18$ & $33.53 \pm 20$ & $5.64 \pm 0.001$ & $6.76 \pm 0.99$ & $0.007^{*}$ \\
\hline IL-7 & 1.1 & $15 \pm 1.96$ & $53.07 \pm 39.57$ & $16.99 \pm 0.001$ & $16.33 \pm 1.78$ & 0.502 \\
\hline IL-12 & 3.5 & $10.14 \pm 3.37$ & $84.98 \pm 70.64$ & $3.38 \pm 0.001$ & $13.36 \pm 5.96$ & 0.383 \\
\hline IL-17 & 3.3 & $1.65 \pm 0.00$ & $3.22 \pm 0.81$ & $1.65 \pm 0.00$ & $1.65 \pm 0.00$ & 0.374 \\
\hline \multicolumn{7}{|c|}{ Anti-inflammatory } \\
\hline IL-4 & 0.7 & $0.84 \pm 0.22$ & $1.03 \pm 0.26$ & $1.03 \pm 0.001$ & $0.92 \pm 0.19$ & 0.909 \\
\hline IL-5 & 0.6 & $2.12 \pm 0.50$ & $7.16 \pm 4.04$ & $2.45 \pm 0.001$ & $2.79 \pm 0.62$ & 0.327 \\
\hline IL-13 & 0.7 & $2.43 \pm 0.15$ & $27.18 \pm 22.83$ & $2.37 \pm 0.001$ & $3.27 \pm 0.51$ & 0.38 \\
\hline IL-10* & 0.3 & $6.10 \pm 0.32$ & $74.66 \pm 46.85$ & $2.06 \pm 0.003$ & $7.81 \pm 4.04$ & $<0.0001^{*}$ \\
\hline TGF- $\beta 1$ & 1.7 & $13.77 \pm 4.58$ & $37.85 \pm 17.47$ & $187.59 \pm 0.01$ & $33 \pm 16.83$ & $<0.0001$ \\
\hline TGF- $\beta 2$ & 14.7 & $40.33 \pm 9.62$ & $59.44 \pm 8.29$ & $86.71 \pm 0.01$ & $69.79 \pm 9.06$ & 0.008 \\
\hline TGF- $\beta 3$ & 2.8 & $1.4 \pm 0.00$ & $4.30 \pm 1.19$ & $1.4 \pm 0.00$ & $4.55 \pm 1.64$ & 0.013 \\
\hline
\end{tabular}

SEM: standard error of the mean (in pg/ml). VVC+: presence of vulvovaginal candidiasis infection; VVC-: absence of vulvovaginal candidiasis infection; HIV: human immunodeficiency virus; IL = interleukin; MIP = macrophage inflammatory protein; TGF: transforming growth factor. IFN = interferon; G-CSF = granulocyte colony-stimulating factor; GM-CSF = granulocyte-macrophage colony-stimulating factor. * Mann-Whitney $U$ nonparametric tests; ${ }^{* *}$ Kruskal-Wallis nonparametric tests.

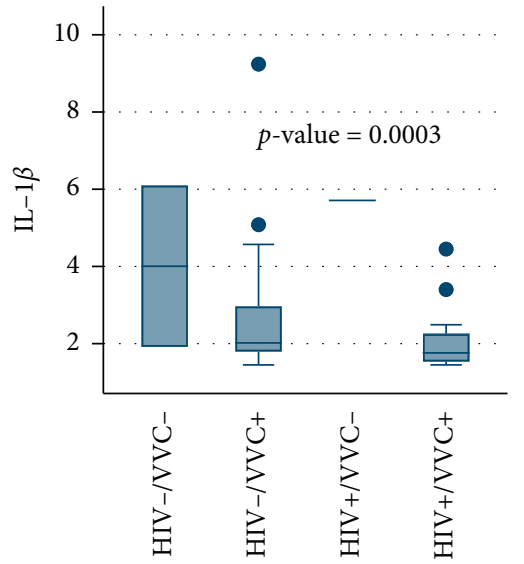

(a)

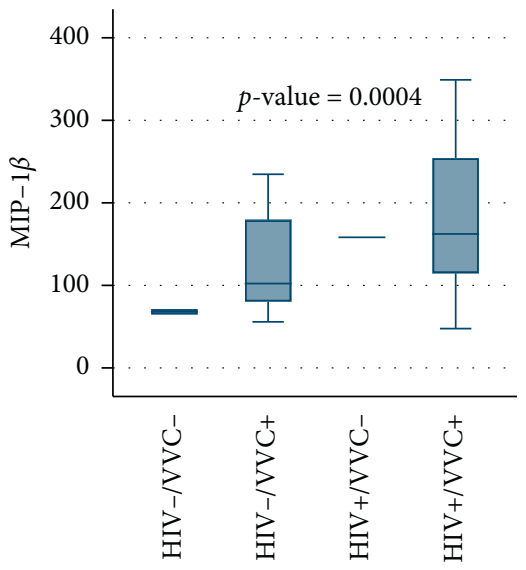

(b)

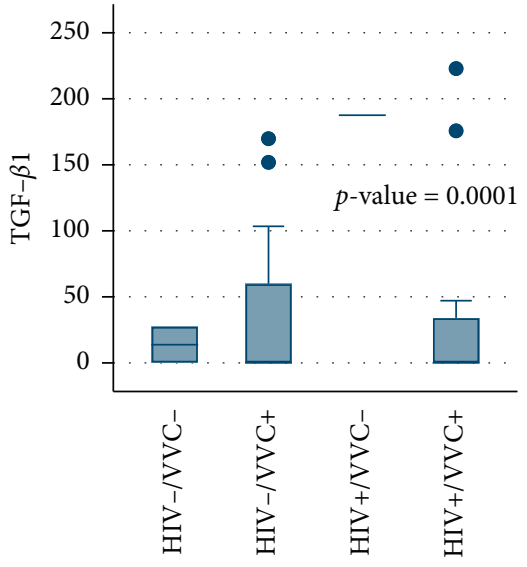

(c)

FIgUre 1: Continued. 


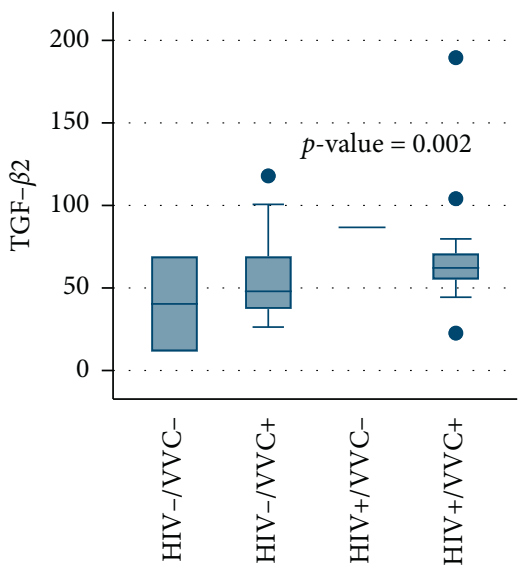

(d)

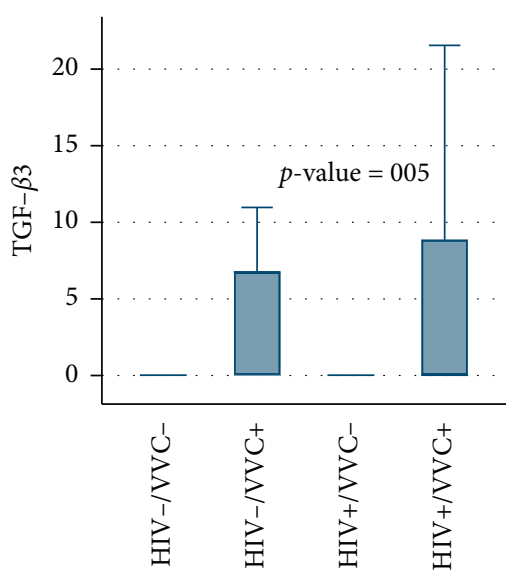

(e)

Figure 1: Multiple comparisons of the mean concentrations of (a) IL- $1 \beta$, (b) MIP-1 $\beta$, (c) TGF- $\beta 1$, (d) TGF- $\beta 2$, and (e) TGF- $\beta 3$ using Bonferroni post hoc tests.

TABLE 2: Wilks' lambda values.

\begin{tabular}{|c|c|c|c|c|}
\hline Test of functions & Wilks' lambda & Chi-square test & $\mathrm{df}$ & $p$ value \\
\hline 1 through 3 & 0.116 & 95.78 & 9 & $<0.0001$ \\
\hline 2 through 3 & 0.525 & 28.694 & 4 & $<0.0001$ \\
\hline 3 & 0.978 & 0.978 & 1 & 0.323 \\
\hline
\end{tabular}

TABle 3: Classification results: functions' coefficients.

\begin{tabular}{lcccc}
\hline & & \multicolumn{2}{c}{ Predicted group membership } \\
& HIV-VVC- & HIV-VVC+ & HIV + VVC- & HIV + VVC+ \\
\hline Predictor variables & & & 0.084 & -0.028 \\
TGF- $\beta 1$ & -0.003 & -0.016 & -0.345 & 0.792 \\
TGF- $\beta 3$ & 0.135 & 0.598 & 0.027 & 0.072 \\
MIP-1 $\beta$ & 0.022 & 0.051 & -11.41 & -9.249 \\
Constant & -2.113 & -5.683 & & \\
\hline
\end{tabular}

0.93 Mahalanobis distance units away from the $\mathrm{HIV}+\mathrm{VVC}+$ group, TGF- $\beta 3$ was 3.271 Mahalanobis distance units away from the HIV-VVC + group, and TGF- $\beta 1$ was 2.703 Mahalanobis distance units away from the $\mathrm{HIV}+\mathrm{VVC}+$ group. These findings are supported by findings in Table 3 that showed MIP- $1 \beta$ and TGF- $\beta 3$ as significantly predicting the HIV + VVC + group while TGF$\beta 1$ predicting the HIV-VVC+ group.

\subsection{Concentrations of Genital Cytokines/Chemokines Asso-} ciated with Increased Odds for Symptomatic Vulvovaginal Candidiasis in HIV-Infected and Uninfected Women. In univariate analysis, of the 20 cytokines/chemokines measured from cervicovaginal secretions, mean levels of IL-2, IL-4, IL-5, IL-6, IL-7, IL-8, IL-12, IL-13, IL-17, G-CSF, GM-CSF, IFN- $\gamma$, MCP-1, and TNF- $\alpha$ were normally distributed and showed significant differences across the groups using ANOVA (Table 5).
After adjusting for multiple comparisons using post hoc Bonferroni pairwise tests, mean concentrations of IL-2, IL-4, IL-5, IL-6, IL-7, IL-8, IL-12, IL-13, IL-17, G-CSF, and MCP1 were confirmed to display significant differences across the study groups. With the exception of the proinflammatory chemokine IL-8 that had higher mean genital levels in the HIV-VVC + group, all other proinflammatory (IL-6, G-CSF, and MCP-1), Th1 (IL-2 and IL-12), Th2 (IL-4, IL-5, and IL-13), and Th17 (IL17) cytokines displayed higher mean levels in the HIV + VVC + group.

Furthermore, after performing the canonical discriminant analysis from all normally distributed variables that displayed significant univariate associations, only one canonical function composed of one cytokine (IL-13), an immune-allergic mediated anti-inflammatory cytokine, was shown to significantly predict a case classification into $\mathrm{HIV}+\mathrm{VVC}+$ group. That one discriminant function had eigenvalue of 0.48 with a canonical correlation of 0.57 . This function contributed $100 \%$ of the total variance: Box's M 


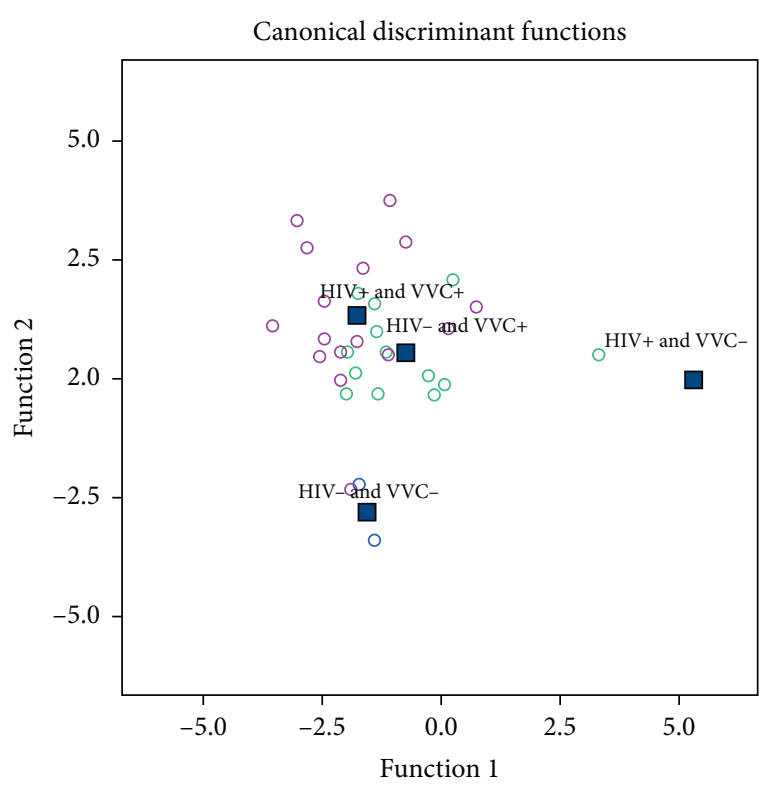

HIV/VVC status

- HIV- and VVC-

- HIV- and VVC+

HIV+ and VVC-

Figure 2: Canonical discriminant functions displaying centroid groups: 4 study groups (HIV-VVC-; HIV-VVC+; HIV + VVC; and $\mathrm{HIV}+\mathrm{VVC}+$ ) discriminated by $2 \mathrm{CDA}$ functions made of 3 significant variates (MIP- $1 \beta$, TGF- $\beta 1$, and TGF- $\beta 3$ ) which contributed $99.5 \%$ of the total variance.

TABle 4: Predictor variables displaying maximum Mahalanobis distances between the two closest groups (HIV-VVC+ and $\mathrm{HIV}+\mathrm{VVC}+$ ).

\begin{tabular}{lcccc}
\hline & \multicolumn{4}{c}{ Mahalanobis distances ( $D$-squared) } \\
& \multicolumn{4}{c}{ Exact $F$} \\
\hline Predictor variables & SD value & df1 & df2 & $p$ value \\
MIP-1 $\beta$ & 0.93 & 1 & 45 & 0.34 \\
TGF- $\beta 3$ & 3.271 & 2 & 44 & 0.047 \\
TGF- $\beta 1$ & 2.703 & 3 & 43 & 0.05 \\
\hline
\end{tabular}

(2.063), $p \quad(0.37)>\alpha \quad(0.001) ; \quad$ Wilks' lambda $=.677$, $\chi^{2}=17.76 ; p<0.0001$.

\section{Discussion}

Findings from this study showed that more than one pathogenic mechanism can explain the occurrence of symptomatic VVC in HIV-infected women.

4.1. New Insights about Treg and Th17 Cells in HIV-Infected Women with Symptomatic VV. Using discriminant analysis, it was found that mean levels of circulatory TGF- $\beta 3$ were significantly higher in women with VVC regardless of their HIV serostatus. In addition, after adjusting for multiple comparisons using post hoc Bonferroni pairwise tests, mean concentrations of genital IL-6, IL-8, IL-10, IL-17, and TGF$\beta 3$ were significantly higher in HIV-infected women coinfected with VVC. Previous studies have shown that, in the presence of TGF- $\beta 3$ and IL- 6 , naïve $C D+T$ cells will differentiate into Th17 cells [12-16]. Newly differentiated Th17 cells will then subsequently produce more IL- 6 in addition to IL-8 and IL-17 as effector cytokines [12-16]. These authors have also shown that functional Th17 cells were able to produce IL-21 and IL-23 as effector cytokines [12-16], but these cytokines were not measured in this present study.

As a major effector cytokine for Th17 functionality, IL-17 induces a strong inflammatory response via IL- 6 and IL-8 [11-15]. Besides inflammation, IL-6 in return is involved in regulating acute-phase and immune responses as well as hematopoietic functions [17]. On the other hand, IL-8 has a strong chemotactic activity for neutrophils at the site of the infection in addition to the recruitment and activation of other cells such as NK cells and GM-CSF [18].

The production of classical Th17 cells obtained via the presence of TGF- $\beta$ favors subsequent production of higher levels of IL-10 [12-16], as also shown in our present study. Furthermore, the same authors have demonstrated that, in the presence of TGF- $\beta$ and IL-10, CD4+ T cells will differentiate to regulatory $\mathrm{T}$ cells (Treg cells) resulting in the production of more IL-10 that blocks Th17-mediated inflammation in return [12-16]. From these findings of the previous authors and our own findings, we can then hypothesize that the obtained Treg cells via IL-10 represent regulatory mechanisms in order to prevent excessive tissue damage from Th17 cells via IL-6 and IL-8, suggesting the existence of a new paradigm of Th17/Treg mechanisms in HIV infected women with VVC.

In this study, although some Th1 cytokines (IL-2 and IL-12) were significantly higher among HIV-infected women with VVC, IFN- $\gamma$, which is known to be a major Th1 effector cytokine, was shown to be significantly low. Liu et al. have shown that when myeloid cells are infected, there is a strong production of IL-12 that subsequently leads to the differentiation of Th1 cells, hence the production of IFN- $\gamma$ [19]. However, during some studies on Th1-inducing effector cytokines, it was reported that, during various pathological conditions, the role played by IFN $-\gamma$ was not critically important as compared to IL- 12 $[14,20]$. These discrepant findings regarding CD4-mediated immune responses were only reconciled after Th17 cells were discovered [14]. It became evident that IL-12 and IL-23 share the $\mathrm{p} 40$ subunit of IL- 12 , and also, IL-12R $\beta 1$ is a subunit of IL-23R [21]. This was subsequently confirmed by animal studies that showed that mice lacking these IL-12 associated subunits were deficient to both IL-12 (Th1 effector cytokine) and IL-23 (Th17 effector cytokine) [21].

In conclusion, there is $T h 1 / T h 2$ paradigm shift in the pathogenesis of VVC among HIV infected women [12-16]. Of major importance was the observed significant level of TGF- $\beta 3$, a member of TGF- $\beta$ family of cytokines. It is well established that the immunoregulatory properties of these cytokines are associated with the absence of anti-Candida cell-medicated immunity in the vagina [22-24]. In addition, Th17 cells rather than Th1/Th2 paradigms were demonstrated to be key players for immune responses in HIV 
TABLE 5: Comparisons of cervicovaginal secretions' mean levels (in pg/ml) of cytokine distribution across the study groups.

\begin{tabular}{|c|c|c|c|c|c|c|}
\hline & & \multicolumn{5}{|c|}{ Study group } \\
\hline & & $\begin{array}{c}\mathrm{HIV}-\text { and VVC- } \\
\text { mean } \pm \text { SEM< }\end{array}$ & $\begin{array}{l}\mathrm{HIV}-\text { and VVC+ } \\
\text { mean } \pm \mathrm{SEM}\end{array}$ & $\begin{array}{l}\mathrm{HIV}+\text { and VVC- } \\
\text { mean } \pm \text { SEM }\end{array}$ & $\begin{array}{c}\mathrm{HIV}+\text { and VVC+ } \\
\text { mean } \pm \text { SEM }\end{array}$ & $\begin{array}{l}\text { ANOVA } p \\
\text { value }\end{array}$ \\
\hline $\begin{array}{l}\text { Cytokine } \\
\text { types }\end{array}$ & \multicolumn{6}{|c|}{ Cytokine limit of detection $(\mathrm{pg} / \mathrm{mL})$} \\
\hline \multicolumn{7}{|c|}{ Proinflammatory } \\
\hline G-CSF & 1.7 & $3.82 \pm 1.3$ & $14.075 \pm 3.42$ & $0.85 \pm 0.00$ & $21.894 \pm 5.62$ & 0.002 \\
\hline GM-CSF & 2.2 & $42.24 \pm 2.55$ & $57.81 \pm 3.59$ & $42.34 \pm 0.001$ & $57.98 \pm 5.34$ & 0.007 \\
\hline IFN- $\gamma$ & 6.4 & $7.148 \pm 3.63$ & $29.206 \pm 8.62$ & $3.91 \pm 0.001$ & $55.62 \pm 20.035$ & 0.037 \\
\hline $\mathrm{IL}-2$ & 1.6 & $0.8 \pm 0.00$ & $4.795 \pm 1.81$ & $0.80 \pm 0.00$ & $6.77 \pm 2.12$ & 0.022 \\
\hline IL-8 & 1 & $26.486 \pm 8.156$ & $494.55 \pm 148.95$ & $10.84 \pm 0.001$ & $320.67 \pm 97.65$ & 0.004 \\
\hline TNF- $\alpha$ & 6 & $13.106 \pm 6.48$ & $44.079 \pm 10.04$ & $14.07 \pm 0.001$ & $56.32 \pm 11.09$ & 0.003 \\
\hline MCP-1 & 1.1 & $2.35 \pm 0.34$ & $8.03 \pm 2.23$ & $2.32 \pm 0.001$ & $13.21 \pm 4.27$ & 0.037 \\
\hline IL- $1 \beta^{* *}$ & 0.6 & $127.61 \pm 59.79$ & $1975.77 \pm 779.89$ & $143.11 \pm 0.001$ & $2181.43 \pm 803.23$ & $0.001^{* *}$ \\
\hline IL-7 & 1.1 & $0.55 \pm 0.00$ & $0.963 \pm 0.31$ & $0.57 \pm 0.00$ & $1.076 \pm 0.3$ & 0.012 \\
\hline MIP- $1 \beta$ & 2.4 & $8.86 \pm 2.39$ & $12.23 \pm 3.43$ & $8.13 \pm 0.001$ & $16.67 \pm 3.95$ & 0.235 \\
\hline IL-12 & 3.5 & $1.916 \pm 0.58$ & $3.127 \pm 0.71$ & $1.75 \pm 0.00$ & $4.15 \pm 0.82$ & 0.001 \\
\hline IL-17 & 3.3 & $5.799 \pm 2.27$ & $19.003 \pm 4.34$ & $1.95 \pm 0.001$ & $20.53 \pm 4.33$ & 0.002 \\
\hline IL-6 & 2.6 & $1.911 \pm 0.548$ & $5.582 \pm 1.35$ & $1.38 \pm 0.001$ & $11.583 \pm 3.74$ & 0.017 \\
\hline \multicolumn{7}{|c|}{ Anti-inflammatory } \\
\hline IL-4 & 0.7 & $0.59 \pm 0.18$ & $2.062 \pm 0.44$ & $0.62 \pm 0.001$ & $2.27 \pm 0.46$ & 0.003 \\
\hline IL-5 & 0.6 & $0.257 \pm 0.09$ & $0.394 \pm 0.057$ & $0.30 \pm 0.00$ & $0.524 \pm 0.08$ & 0.004 \\
\hline IL-13 & 0.7 & $0.83 \pm 0.22$ & $1.56 \pm 0.22$ & $0.47 \pm 0.001$ & $1.78 \pm 0.26$ & $<0.0001$ \\
\hline TGF- $\beta 1^{* *}$ & 1.7 & $4.72 \pm 1.92$ & $4.16 \pm 1.6$ & $6.88 \pm 0.001$ & $1.588 \pm 1.09$ & $0.0024^{* *}$ \\
\hline TGF- $\beta 2 * *$ & 14.7 & $13.034 \pm 1.16$ & $11.96 \pm 1.21$ & $8.39 \pm 0.001$ & $12.52 \pm 2.09$ & $0.012^{* *}$ \\
\hline TGF- $\beta 3^{* *}$ & 2.8 & $2.51 \pm 0.44$ & $8.56 \pm 6.003$ & $1.42 \pm 0.001$ & $2.32 \pm 0.25$ & $0.001^{* *}$ \\
\hline IL-10 & 0.3 & $0.902 \pm 0.078$ & $0.978 \pm 0.09$ & $0.9 \pm 0.001$ & $1.249 \pm 0.17$ & 0.135 \\
\hline
\end{tabular}

SEM: standard error of the mean (in $\mathrm{pg} / \mathrm{ml}$ ). VVC+: presence of vulvovaginal candidiasis infection; VVC-: absence of vulvovaginal candidiasis infection; HIV: human immunodeficiency virus; IL = interleukin; MIP = macrophage inflammatory protein; TGF: transforming growth factor; IFN = interferon; G-CSF = granulocyte colony-stimulating factor; GM-CSF = granulocyte-macrophage colony-stimulating factor. ${ }^{* *}$ Kruskal-Wallis nonparametric tests.

infected women with symptomatic VVC [12-16]. Studies have also shown that, during the course of HIV infection, there are progressive declines of Th17 cells as well as a decrease of Th17/Treg ratio [16]. This unbalance seems to be more important in advanced HIV disease [16]. Most of these studies however have been assessing the loss of Th17 cells in the gastrointestinal mucosa of HIV-infected patients [16]. The role of Th17 cells during Candida infection in different other anatomical sites remains largely unknown. The associated hypothesis to the role played by Th17 cells at the mucosal surfaces of the vagina during Candida infection could be the recruitment of PMN cells, via the presence of IL-8, which can be inflammatory but nonprotective as demonstrated by Fidel Jr. et al. during a human life challenge model of VVC [25]. Whilst our findings are keeping with some previously published studies which have shown that inhibition of Th17 cell differentiation led to decrease in production of IL-17 with consequent exacerbation of VVC, other authors have suggested that inflammatory response during VVC occurs independently of Th17 cell lineage [26-28].

4.2. The Role of IgE-Mediated Hypersensitivity Response. Findings from this study showed that Th2-type cytokines (IL-4, IL-5, and IL-13) were significantly increased in VVC women coinfected with HIV. Previous analyses of Th2 cytokines in clinical studies showed only a small amount of IL-4, IL-5, and IL-13 in vaginal fluid with no effect on fungal burden [6]. However, controversies were raised by Babula et al. who showed that women with recurrent VVC had increased vaginal levels of IL-4 polymorphism, possibly resulting in reduced production of anticandidal compounds such as nitric oxide and mannose-binding lectin (MBL) [29]. A similar controversy from the literature was from a study that showed that increased IL- 4 and IL-10 were associated with higher fungal burden and VVC severity $[30,31]$. The authors have then hypothesized that the associations between recurrent VVC and the vaginal presence of significant levels of IL-4, IL-5, and IL-13 could be the result of an allergic reaction due to the presence of Candida-specific IgE production and eosinophil stimulation in HIV infected women [30, 31]. Moreover, a study by Yano et al. (2012) has shown that the vaginal S100 alarmin response to Candida does not require Th17 cell lineage and effector cytokines, suggesting that the inflammatory response during VVC may occur independently of the Th17 pathway [27].

Therefore, in addition to the hypothesized Th17 altered immune response as the pathogenic mechanisms in VVC patients coinfected with HIV, the role played by local hypersensitivity cannot be neglected. Our cohort of HIV infected women with symptomatic VVC had significantly higher levels of IL-4, IL-5, and IL-13 in their genital 
secretions, suggestive of a strong hypersensitivity response. In any case, the hypothesis that VVC in HIV infected women can be a result of a local hypersensitivity reaction might be relevant to only a group of women with some predisposing factors. A further study is warranted in which genital $\operatorname{IgE}$ levels are explored in HIV infected women with symptomatic VVC in order to support our results.

4.3. The Role of HIV-Induced Severe Immunosuppression. Although we did not consider patients' CD4+ T cell count values when performing canonical discriminant analysis, all $26 \mathrm{HIV}$ infected patients had either advanced (CD4+ T count 200-349 cells $/ \mathrm{mm}^{3}$ ) or severe immunosuppression (CD4+ T count $<200$ cells $/ \mathrm{mm}^{3}$ ). Our previous reports using the same cohort of women have shown that HIV infected women whose CD4+ T cells were less than 200/ $\mathrm{mm}^{3}$ had more than 7-fold high chance of developing VVC as compared to their HIV-negative counterparts as well as HIV-infected women whose CD4+ T cells are above 200 [8]. However, when comparing only HIV-infected women stratified by levels of immunosuppression, the odds increased by 9 -fold and 60-fold higher for women with advanced and severe immunosuppression, respectively [11].

\section{Conclusions and Limitations}

In HIV-infected women, VVC was primarily and strongly predicted by the presence of circulatory and genital TGF- $\beta 3$, a key factor for Th17 differentiation in the presence of IL-6, which can also differentiate into Treg cells and becomes a strong downregulatory and anti-inflammatory response in the presence of IL-10. In addition, the roles played by Candida-induced inflammatory but nonprotective Th17 induced responses in the lower genital tract should not be ignored. Secondary pathogenic mechanisms included the presence of vaginal IL-13, an allergic mediated Th2 cytokine, and HIV-induced severe immunosuppression. The latter was supported by the presence of circulatory MIP-1 $\beta$, a marker of chronic immune activation observed during severe $\mathrm{HIV}$-induced immunosuppression.

The limitation of this study is its cross-sectional design. Although our findings on cytokine profiles are suggestive of Th17/Treg responses, other cell types such as NK cells or macrophages could have also secreted similar cytokines, and the final confirmation should come from measuring cytokine profiles from post-T cell stimulation and from culture supernatants. Nevertheless, we cannot neglect the value of the obtained findings on cytokines from clinical specimens since they provide predictive clinical information and insights into disease mechanisms. In addition, the performed discriminant function analysis did not take into account CD4 counts of the study participants. The two closest centroid groups were $\mathrm{HIV}+\mathrm{VVC}+$ and $\mathrm{HIV}-\mathrm{VVC}+$. It is possible that $\mathrm{HIV}+\mathrm{VVC}+$ women on HAART with $\mathrm{CD} 4$ count closer to 350 cells $/ \mathrm{mm} 3$ could have an immune response relatively comparable to the $\mathrm{HIV}-\mathrm{VVC}$ + group, thus bringing group centroids for the two study groups closer. IgE and eosinophil levels in the genital tract of women were not measured in order to back up the theory of hypersensitivity. Raw data for this manuscript can be obtained on request.

\author{
Abbreviations \\ ART: Antiretroviral therapy \\ BV: Bacterial vaginosis \\ DA: Discriminant analysis \\ HIV: Human immunodeficiency virus \\ LGTIs: Lower genital tract infections \\ NHLS: National Health Laboratory Services \\ NRTIs: Nucleoside reverse-transcriptase inhibitors \\ NNRTI: Nonnucleoside reverse-transcriptase inhibitor \\ PHC: Primary healthcare \\ PI: $\quad$ Protease inhibitor \\ PMN: Polymorphonuclear neutrophils \\ RVVC: Recurrent vulvovaginal candidiasis \\ Th17: T helper 17 \\ Treg: $\quad$ Regulatory T cells \\ VL: $\quad$ Viral load \\ VVC: Vulvovaginal candidiasis \\ WSU: Walter Sisulu University.
}

\section{Data Availability}

The datasets used and/or analyzed during the current study are available from the corresponding author on reasonable request.

\section{Ethical Approval}

Ethical approval was obtained from the Biomedical Research Ethics Committee of the University of KwaZulu-Natal (ref. BE 224/11). Confidentiality was maintained throughout the study. Minor patients were excluded as mentioned before. Permissions to conduct the study were obtained from the clinic management. In order to protect privacy and confidentiality of patients, no names were recorded, and instead, a personalized research number was used for each patient, and only investigators had access to the collected data.

\section{Consent}

All patients signed written informed consent.

\section{Conflicts of Interest}

The authors declare that they have no conflicts of interest.

\section{Authors' Contributions}

TA conceived the study and its design, collected the data, participated in data analysis, and wrote the study proposal and the final draft of this manuscript. BL performed data analysis and interpretation and edited the final draft of the manuscript. 


\section{Acknowledgments}

The authors express their gratitude to the laboratory staff, Immunology Department, National Health Laboratory Services at the University of Pretoria, for their assistance in the use of Luminex.

\section{References}

[1] L. De Repentigny, D. Lewandowski, and P. Jolicoeur, "Immunopathogenesis of oropharyngeal candidiasis in human immunodeficiency virus infection," Clinical Microbiology Reviews, vol. 17, no. 4, pp. 729-759, 2004.

[2] P. L. Fidel Jr., "Candida-host interactions in HIV disease: relationships in oropharyngeal candidiasis," Advances in Dental Research, vol. 19, no. 1, pp. 80-84, 2006.

[3] P. L. Fidel Jr., "History and new insights into host defense against vaginal candidiasis," Trends in Microbiology, vol. 12, no. 5, pp. 220-227, 2004.

[4] B. N. Taylor, M. Saavedra, and P. L. F. Jr Jr, "Local Th1/Th2 cytokine production during experimental vaginal candidiasis: potential importance of transforming growth factor- $B$," Medical Mycology, vol. 38, no. 6, pp. 419-431, 2000, Epub 2001/02/24.

[5] B. L. Shacklett and P. A. Anton, "HIV infection and gut mucosal immune function: updates on pathogenesis with implications for management and intervention," Current Infectious Disease Reports, vol. 12, no. 1, pp. 19-27, 2010.

[6] M. M. Barousse, B. J. Van Der Pol, D. Fortenberry, D. Orr, and P. L. Fidel Jr, "Vaginal yeast colonisation, prevalence of vaginitis, and associated local immunity in adolescents," Sexually Transmitted Infections, vol. 80, no. 1, pp. 48-53, 2004.

[7] L. A. Bergmeier and T. Lehner, "Innate and adaptive mucosal immunity in protection against HIV infection," Advances in Dental Research, vol. 19, no. 1, pp. 21-28, 2006.

[8] T. Apalata, B. Longo-Mbenza, W. A. Sturm, W. H. Carr, and P. Moodley, "Expression of toll-like receptor (TLR)-2 and TLR4 in monocytes following stimulations by genital secretions of HIV infected and uninfected women with symptomatic vulvo-vaginal candidiasis," International Journal of Medicine and Medical Sciences, vol. 6, no. 5, pp. 134-139, 2014.

[9] T. Apalata, B. Longo-Mbenza, A. Sturm, W. Carr, and P. Moodley, "Factors associated with symptomatic vulvovaginal candidiasis: a study among women attending a primary healthcare clinic in Kwazulu-Natal, South Africa," Annals of Medical and Health Sciences Research, vol. 4, no. 3, pp. 410416, 2014.

[10] J. Sherrard, G. Donders, D. White, and J. S. Jensen, "European (IUSTI/WHO) guideline on the management of vaginal discharge, 2011," International Journal of STD \& AIDS, vol. 22, no. 8, pp. 421-429, 2011, Epub 2011/07/29.

[11] T. Apalata, W. H. Carr, W. A. Sturm, B. Longo-Mbenza, and P. Moodley, "Determinants of symptomatic vulvovaginal candidiasis among human immunodeficiency virus type 1 infected women in rural KwaZulu-Natal, South Africa," Infectious Diseases in Obstetrics and Gynecology, vol. 2014, Article ID 387070, 2014.

[12] J. Yano, M. C. Noverr, and P. L. Fidel Jr, "Cytokines in the host response to Candida vaginitis: identifying a role for nonclassical immune mediators, S100 alarmins," Cytokine, vol. 58, no. 1, pp. 118-128, 2012.

[13] D. R. Littman and A. Y. Rudensky, "Th17 and regulatory $\mathrm{T}$ cells in mediating and restraining inflammation," Cell, vol. 140, no. 6, pp. 845-858, 2010.
[14] N. Hernández-Santos and S. L. Gaffen, "Th17 cells in immunity to Candida albicans," Cell Host \& Microbe, vol. 11, no. 5, pp. 425-435, 2012.

[15] S. Cypowyj, C. Picard, L. Maródi, J.-L. Casanova, and A. Puel, "Immunity to infection in IL-17-deficient mice and humans," European Journal of Immunology, vol. 42, no. 9, pp. 22462254, 2012, Epub 2012/09/06.

[16] S. L. Bixler and J. J. Mattapallil, "Loss and dysregulation of Th17 cells during HIV infection," Journal of Immunology Research, vol. 2013, Article ID 852418, 9 pages, 2013.

[17] T. Kishimoto, "IL-6: from its discovery to clinical applications," International Immunology, vol. 22, no. 5, pp. 347-352, 2010.

[18] F. Lin, C. M.-C. Nguyen, S.-J. Wang, W. Saadi, S. P. Gross, and N. L. Jeon, "Effective neutrophil chemotaxis is strongly influenced by mean IL-8 concentration," Biochemical and Biophysical Research Communications, vol. 319, no. 2, pp. 576-581, 2004.

[19] J. Liu, S. Cao, S. Kim et al., "Interleukin-12: an update on its immunological activities, signaling and regulation of gene expression," Current Immunology Reviews, vol. 1, no. 2, pp. 119-137, 2005.

[20] L. Steinman, "A brief history of TH17, the first major revision in the TH1/TH2 hypothesis of T cell-mediated tissue damage," Nature Medicine, vol. 13, no. 2, pp. 139-145, 2007.

[21] N. Ghilardi and W. Ouyang, "Targeting the development and effector functions of TH17 cells," Seminars in Immunology, vol. 19, no. 6, pp. 383-393, 2007.

[22] D. M. Leblanc, M. M. Barousse, and P. L. Fidel Jr., "Role for dendritic cells in immunoregulation during experimental vaginal candidiasis," Infection and Immunity, vol. 74 , no. 6 , pp. 3213-3221, 2006.

[23] J. Kosonen, A. Rantala, C. H. Little et al., "Increased levels of Candida albicans mannan-specific T-cell-derived antigen binding molecules in patients with invasive candidiasis," Clinical and Vaccine Immunology, vol. 13, no. 4, pp. 467-474, 2006.

[24] L. Romani, "Innate and adaptive immunity in Candida albicans infections and saprophytism," Journal of Leukocyte Biology, vol. 68, no. 2, pp. 175-179, 2000.

[25] P. L. Fidel Jr., M. Barousse, T. Espinosa et al., "An intravaginal live Candida challenge in humans leads to new hypotheses for the immunopathogenesis of vulvovaginal candidiasis," Infection and Immunity, vol. 72, no. 5, pp. 2939-2946, 2004.

[26] M. Zahra Talaei, S. Sheikhbahaei, V. Ostadi et al., "Recurrent vulvovaginal candidiasis: could it Be related to cell-mediated immunity defect in response to candida antigen?" International Journal of Fertility and Sterility, vol. 11, no. 3, pp. 134-141, 2017.

[27] J. Yano, J. K. Kolls, K. I. Happel, F. Wormley, K. L. Wozniak, and P. L. Fidel Jr, "The acute neutrophil response mediated by S100 alarmins during vaginal Candida infections is independent of the Th17-pathway," PLoS One, vol. 7, no. 9, Article ID e46311, 2012.

[28] W. Mendling and U. Koldovsky, "Investigations by cellmediated immunologic tests and therapeutic trials with thymopentin in vaginal mycoses," Infectious Diseases in Obstetrics and Gynecology, vol. 4, no. 4, pp. 225-231, 1996.

[29] O. Babula, G. Lazdane, J. Kroica, I. M. Linhares, W. J. Ledger, and S. S. Witkin, "Frequency of interleukin-4 (IL-4) -589 gene polymorphism and vaginal concentrations of IL-4, nitric oxide, and mannose-binding lectin in women with recurrent vulvovaginal candidiasis," Clinical Infectious Diseases, vol. 40, no. 9, pp. 1258-1262, 2005. 
[30] T. Belay, F. O. Eko, G. A. Ananaba et al., "Chemokine and chemokine receptor dynamics during genital chlamydial infection," Infection and Immunity, vol. 70, no. 2, pp. 844-850, 2002.

[31] G. Fischer, "Chronic vulvovaginal candidiasis: what we know and what we have yet to learn," Australasian Journal of Dermatology, vol. 53, no. 4, pp. 247-254, 2012. 